\title{
The Role of E-cadherin in the Differentiation of Ductal and Lobular Breast Carcinomas
}

Tara M A Saeed $(\mathrm{MSc})^{1}$, Tamara Almufty $(\mathrm{FICM})^{2}$, Fairuz A Kakasur $(\mathrm{MSc})^{3}$ and Namir Ghanim Al-Tawil Hawler (FICMS/CM/FFPH) ${ }^{4}$

\begin{abstract}
\end{abstract}
Background: Diagnosis of certain types of breast cancer can be difficult due to similarities in their histopathological patterns. In such cases markers can be useful in confirming results. E-cadherin is one such marker.

Objective: To differentiate the rate of E-cadherin immunohistochemical expression between the major histological types of breast cancer (lobular versus ductal), in addition to correlating it to the clinicopathological factors such as age of patients and grade of the tumor and explore the effectiveness of using it in distinguishing the cases with overlapping features.

Patients and Methods: A total of 100 malignant breast samples (55 ductal lesions, 24 lobular lesions, and 21 uncertain types), collected during the period of $2014-2017$ in Erbil, and were reviewed before undergoing E-cadherin immunohistochemistry (IHC) staining. The results from IHC were compared with the results from the morphological study of the samples and they were statistically analyzed in relation with clinicopathological factors. Results: The normal ductal cells had strong complete homogenous membrane E-cadherin reactivity in $85 \%$ of the ductal carcinoma cases. The complete absence of E-cadherin membrane staining occurred in all 24 cases of lobular cases. The rest of the 21 cases, showed variable degrees of staining.

Conclusion: There is diagnostic usefulness in the detection of E-cadherin expression by immunohistochemistry as a tool in distinguishing ductal from lobular carcinoma.

Keywords: Ductal carcinoma, Lobular carcinoma, E-cadherin, Breast.

Corresponding Author: tara.muhammed@hmu.edu.krd

Received: $12^{\text {th }}$ January 2020

Accepted: $23^{\text {th }}$ August 2020

DOI:https://doi.org/10.26505/DJM.19015250112

\footnotetext{
${ }^{1,4}$ College of Medicine- Hawler Medical University-Erbil - Iraq

${ }^{2}$ Maternity Hospital- Erbil - Iraq

${ }^{3}$ Rizgary Hospital- Erbil - Iraq
}

\section{Introduction}

Breast cancer is one of the most common human neoplasms, accounting for approximately one-quarter of all cancers in females. It is said to be associated with western lifestyle, and incidence rates are therefore highest in countries with advanced 
economies, and over one million cases are reported annually worldwide [1]. In addition, a gradual trend is forming for the cancer to occur in females of a younger age and the morbidity rate is increasing, the precise mechanism is presently unclear, but growing evidence has suggested that the tumor microenvironment serves a role in the development of cancer and its prognosis [2]. The incidence of breast carcinoma is $458 / 2381$ in the female population of Erbil [3].

Infiltrating ductal carcinoma (IDC) is reported in the majority of cases, while infiltrating lobular carcinoma (ILC) is approximately one tenth. However the incidence of ILC is increasing, so its characteristics are studied and evaluated clinically. Despite the many similarities, some clinical follow-up data and the patterns of metastasis suggest that ILC and IDC are biologically distinct, and can differ histologically. But controversy still surrounds the prognoses of both types, with ILC having the same, better or even worse than that of IDC $[4 ; 5]$.

The difficulty in distinguishing lobular from ductal carcinoma of the breast, lies in having histological criteria that are equivocally expressed with patterns of diffuse infiltration. This has been solved by immunohistochemical markers, used as tools both individually or in groups in characterizing different cellular proteins including surface or intracellular types [6;7]. Depending on their distribution in different tissues, cadherins are classified into epithelial, neural, and placental. E-cadherin (epithelial) is a glycoprotein of $120 \mathrm{kDa}$, with different domains located extracellularly, and in membrane as well as cytoplasm. This makes it crucial in maintaining mechanical adhesion, cell polarity, stratification, and glandular differentiation, contributing to general architectural configuration of epithelial morphology $[8 ; 9 ; 10]$.

Evidently, with these functions, it was demonstrated that the loss of E-cadherin would initiate the process of tumor formation and growth, entitling E-cadhein to become one of the important tumor suppression proteins $(10 ; 11 ; 12)$. In lobular neoplasia, different types of mutations in E-cadherin gene CDH1 take place, a fact that leads to the molecular deficiency of the extracellular domain of the protein, leading to a defining feature of many infiltrative lobular lesions, having loss of the characteristic membranous E-cadherin staining via immunohistochemistry. Ductal carcinomas, on the other hand, rarely show reduced Ecadherin reactivity, and mostly exhibit clear E-cadherin membranous staining, especially in the well differentiated types $[13 ; 14]$. This study aims to differentiate the phenotypic expressions of E-cadherin between the major histological types of breast cancer (lobular versus ductal), in addition to correlating it to the clinicopathological factors of the tumor and to evaluate the benefits of detecting ECadherin reactions in the cases with uncertain histologies. 


\section{Patients and Methods}

A hundred cases of mammary carcinoma from the local area of Erbil supplied by private labs and public hospitals, were chosen retro- and prospectively, collected over a four-year period between the years 2014 and 2018. Initially, characteristic lesions were defined by morphological criteria and then immunohistochemical (IHC) studies performed. Finally, the score of Ecadherin staining of these cases was used to study the controversial lesions in order to clarify whether the differentiation was ductal or lobular. The slides were reviewed independently by several pathologists in order to establish a diagnosis by determining the morphology, including histological type and grade on the basis of established diagnostic criteria. They were not aware of previous histological diagnosis. After initial histological examination, each case was grouped according to whether the diagnosis was unanimous (agreed upon by both pathologists) or non-unanimous.

Immunohistochemical studies were performed on formalin-fixed paraffinembedded blocks of all 100 cases. Slides were then stained immunohistochemically using antibody to E-cadherin NCH-38 (DAKO), using 4-5 micron thickness sections, deparafinaization, rehydration and epitope target retrieval procedure were performed and incubation with the antibody at (1:100 dilutions) for 60 minutes, Slides were washed in buffer and incubated with link for 10 minutes. After incubation with streptavidin - HRP (LSAB 2 system HRP code K0672) for 10 minutes, slides were developed with diaminobenzidine (liquid $\mathrm{DAB}+$ substrate chromogen system, code K3468) Immunohistochemical staining was visualized, using the Strept AB Complex/horse radish peroxidase (HRP), and finally chromogene labeled signals were studied by three independent pathologists.

\section{Immunohistochemical interpretation}

Clear membranous staining was considered positive. Interpretation by light microscopy was carried out for the immunostaining and scoring. Evaluation of E-Cadherin status was done utilizing a scoring system (15), the correlations of the percentage of positive cells for E-cadherin and their staining intensity: nil for no staining or positivity in the membrane of $<10 \%$ of tumor cells; if $>10 \%$ of tumor cells showed positivity or even had weak/incomplete staining around the membranes, score would be considered 1 ; if $>10 \%$ of tumor cells had a clear complete membranous staining, even if the intensity was weak, it would be considered 2; a strong staining in $>10 \%$ of the membranes of those tumor cells would yield 3. By this scoring system, scores 0 and 1 would be taken as negative, while score 2 would be of weak positivity, and the only strong indicator of positivity is score 3. Staining at areas other than the membranes was excluded from the scoring system, while staining at epithelial cells of the normal ducts were included as positive control.

\section{Statistical analysis}

Data entry and analysis were made by using SPSS version 22. To compare 
proportions, Chi square test of association was used. Statistically significant ' $p$ ' value was considered when $\leq 0.05$.

\section{Results}

\section{Clinicopathological Findings}

A 100 patients with breast cancer sections were evaluated. Their median age was 50 years. The youngest patient was 26 years old while the eldest recorded patient was 84 years old. Half of the patients with ductal and lobular carcinomas (50\%) were above 50 years of age. Fifty five cases were infiltrative ductal carcinomas, twenty four were diagnosed as infiltrative lobular carcinomas. The remaining twenty one cases displayed overlapping morphologies of the two classical histological types, so were designated as query invasive carcinomas (Q). Regardless of the histological phenotype of these tumors, and using Nottingham grading system, the majority of the tumors $(n=78)$ $(78 \%)$ were grade II, $18 \%(n=8)$ were grade III, and only four cases were well differentiated. Table (1) shows that the Ecadherin expression was found in $71.7 \%$ of patients aged less than 50 years, compared with $51.9 \%$ among patients aged $\geq 50$ years, but the difference was not significant $(\mathrm{p}=$ $0.065)$.

Table (1): The relation between age and E-cadherin expression

\begin{tabular}{|c|c|c|c|c|c|c|c|}
\hline \multirow[b]{3}{*}{$\begin{array}{r}\text { Age } \\
\text { (years) }\end{array}$} & \multicolumn{6}{|c|}{ E-cadherin expression } & \multirow[b]{3}{*}{$\mathrm{P}$ value } \\
\hline & \multicolumn{2}{|c|}{ Negative } & \multicolumn{2}{|c|}{ Positive } & \multicolumn{2}{|c|}{ Total } & \\
\hline & $\mathrm{n}$ & $(\%)$ & $\mathrm{N}$ & $(\%)$ & $\mathrm{n}$ & $(\%)^{*}$ & \\
\hline$<50$ & 13 & (28.3) & 33 & (71.7) & 46 & (46.0) & \\
\hline$\geq 50$ & 26 & $(48.1)$ & 28 & $(51.9)$ & 54 & $(54.0)$ & 0.065 \\
\hline Total & 39 & (39.0) & "61 & (61.0) & 100 & (100.0) & \\
\hline
\end{tabular}

It is evident in Table (2) that the more the grade of the tumor, the higher the proportion of patients with positive E-cadherin expression (25\%,
$57.7 \%$, and $72.2 \%$ in grade I, II, III respectively). But the differences were not significant $(\mathrm{p}=$ 0.195).

Table (2): Association between E-cadherin expression and tumor grade

\begin{tabular}{|c|c|c|c|c|c|c|}
\hline & \multicolumn{4}{|c|}{ E-cadherin } & \multirow{3}{*}{ Total } & \multirow{3}{*}{ P value } \\
\hline & \multicolumn{2}{|c|}{ Negative } & \multicolumn{2}{|c|}{ Positive } & & \\
\hline Grade & $\bar{n}$ & $(\%)$ & $\bar{n}$ & $(\%)$ & & \\
\hline$\overline{\mathrm{I}}$ & 3 & $\begin{array}{c}(75.0) \\
\end{array}$ & 1 & $\overline{(25.0)}$ & $\overline{4}$ & 0.195 \\
\hline II & 33 & (43.2) & 45 & $(57.7)$ & 78 & \\
\hline III & 5 & $(27.8)$ & 13 & $(72.2)$ & 18 & \\
\hline$\overline{\text { Total }}$ & 41 & (41.0) & 59 & $\overline{(59.0)}$ & $\overline{100}$ & \\
\hline
\end{tabular}

In the 24 cases of typical ILCs, all (100\%) had lost E-cadherin expression, as in Figure 1(a). Positive E-cadherin expression as in
Figure 1(b) was shown in $85 \%$ of IDC cases (47/55). 


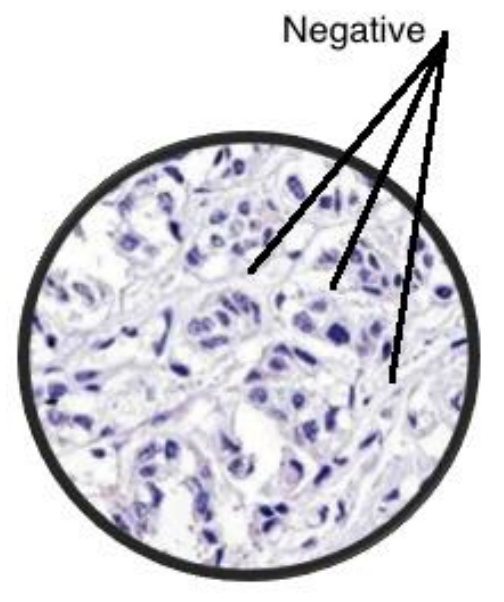

A

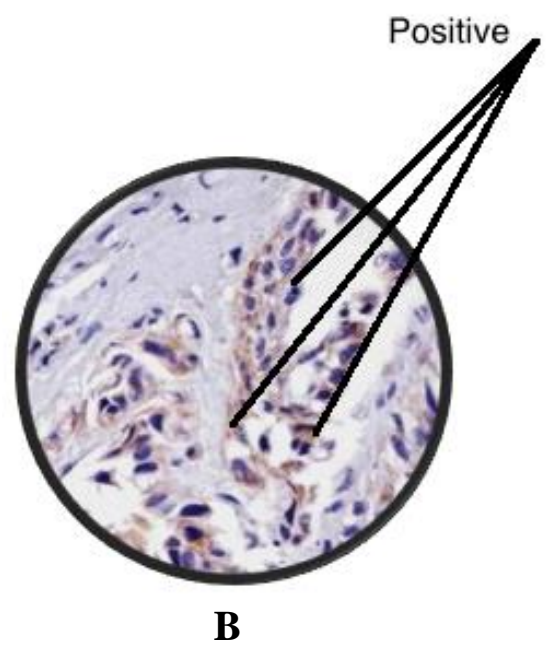

Figure (1) : Ecadherin expression in cells of invasive breast carcinoma. (a) Negative

(b) Positive

It is evident in Table (3) that $70.9 \%$ of the the LC tumors, and 57.1 in the $\mathrm{Q}$ tumors $(\mathrm{p}=$ IDC tumors scored $\geq 3$, compared with $0 \%$ in 0.094 ).

Table (3): E-cadherin staining scores in IDC, ILC, and Q

\begin{tabular}{|c|c|c|c|c|c|c|c|}
\hline \multirow[b]{3}{*}{ Score } & \multicolumn{6}{|c|}{ Tumor types } & \multirow[b]{3}{*}{$\mathrm{P}$ value } \\
\hline & \multicolumn{2}{|c|}{ IDC } & \multicolumn{2}{|c|}{$\mathrm{LC}$} & \multicolumn{2}{|c|}{$\overline{\mathrm{Q}}$} & \\
\hline & No. & $(\%)$ & No. & $(\%)$ & No. & $(\%)$ & \\
\hline 0 & 1 & (1.8) & 20 & $(83.3)$ & 6 & $(28.6)$ & 0.094 \\
\hline $1+$ & 9 & $(16.4)$ & 4 & $(16.7)$ & 2 & $\begin{array}{l}(9.5) \\
\end{array}$ & \\
\hline $2+$ & 6 & $(10.9)$ & 0 & $\begin{array}{l}(0.0) \\
\end{array}$ & 1 & (4.8) & \\
\hline $3+$ & 39 & $(70.9)$ & 0 & $(0.0)$ & 12 & $(57.1)$ & \\
\hline Total & 55 & $(100.0)$ & 24 & $\begin{array}{l}(100.0) \\
\end{array}$ & 21 & $(100.0)$ & \\
\hline
\end{tabular}

Comparing the reactivity of E-cadherin in all significant difference ( $\mathrm{P}$ value $<0.001)$, as in three tumor groups (85\%. 0\%, 61.9\% Table (4). respectively) revealed a statistically

Table (4): E-cadherin staining in IDC, ILC, and QUERY cases

\begin{tabular}{|c|c|c|c|c|c|c|c|c|}
\hline & \multicolumn{6}{|c|}{ E-cadherin Expression } & & \\
\hline & \multicolumn{2}{|c|}{ Positive } & \multicolumn{2}{|c|}{ Negative } & \multicolumn{2}{|c|}{ Mixed } & & \\
\hline & No. & $(\%)$ & No. & $(\%)$ & No. & $(\%)$ & Total $(\mathrm{N})$ & P value \\
\hline$\overline{\mathrm{IDC}}$ & $4 \overline{47}$ & $\begin{array}{l}(85.0) \\
\end{array}$ & 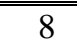 & $\begin{array}{l}(15.5) \\
\end{array}$ & 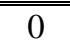 & 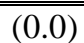 & $\overline{\overline{55}}$ & \\
\hline ILC & $\overline{0}$ & (0.0) & 24 & 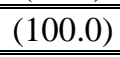 & 0 & $\overline{(0.0)}$ & 24 & $<0.001$ \\
\hline 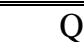 & 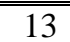 & (61.9) & $\overline{\overline{77}}$ & (33.3) & 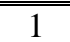 & (4.7) & 21 & \\
\hline Total & 60 & $2(60.0)$ & 39 & (39.0) & 1 & $(1.0)$ & 100 & \\
\hline
\end{tabular}




\section{Discussion}

The main infiltrating carcinomas of the breast are represented by ductal and lobular types. Routinely, histological characteristics are implemented in the differentiation of mammary carcinoma. Sometimes they fail to help in reaching a diagnosis. The difficulty arising from similarity or overlapping features, especially when dealing with invasive lobular carcinoma, is critical due to implications. So the introduction of $\mathrm{E}$ cadherin status helped in the categorization of invasive breast cancer into lobular and ductal types. Differences in E-cadherin staining in infiltrative ductal and infiltrative lobular carcinomas were highly statistically significant $(\mathrm{P}<0.001)$. Our results are similar to those found by Acz and Younes [6; 16]. However one case had foci of both types and was categorized as mixed ductal-lobular type in the same breast.

Out of our hundred cases, pure strong reactivity was seen in $47(85.5 \%)$ cases of histologically proved ductal carcinoma, and were negative in 24(100\%) cases of histologically proved lobular carcinoma as reported by many studies [18]. On the other hand, in 21 cases initially diagnosed as mammary carcinoma with lobular pattern of growth, displaying cellular features of ductal component and typical lobular architectural and cytological features. The possibility of facing difficulties in diagnosis is reported in some studies $[17 ; 19]$. So if their E-cadherin profile shows positivity, this could aid in putting them in a category of IDC with a diffuse cellular pattern similar to that seen in
ILC. 13out of $21(61 \%)$ showed E-cadherin positivity and regarded as IDC whereas the 7 out of the $21(33 \%)$ cases showed E-cadherin negativity regarded as ILC, still one case out of those 21 (4\%) showed truly mixed reactivity. An interesting finding was to have stronger expression of E-cadherin with younger ages, although not highly significant, similar to many other studies stating that it has no relation as an independent factor.

Most of our cases of mammary carcinomas displayed both extremes of E-cadherin expression, score $(3+)$ or score $(0)$ were seen more frequently than other scores. This is reported by ACS et al., as "all or none" phenomenon of E-cadherin expression. Similarly, a variation in E-cadherin intensity was observed in IDCs [15].

Data from literature state a reciprocal correlation between E-cadherin expression and tumor grade. Some reports stated that the reduction in staining could be due to degenerative tumor effect in some IDC of poor differentiation [19]. However some authors do report that there is no correlation with the tumor grade. Other studies explained that some of the reduced expression in IDC could be due to location of the sampled cells in the peripheral regions of ductal carcinomas. They exhibited the features of ILC cells that lost their cohesion and set on an infiltrative pathway. Thus showing loss of E-cadherin expression just like invasive lobular breast cancer $[20 ; 21]$. On the other hand, the finding of very weak expression as 
+1 score in ILC group, noticed in our study, is similar to reports elsewhere, as four of a total of twenty four cases displayed the classic pattern of ILC and demonstrated an abnormal positive E-cadherin expression as dots at the periphery of the nuclei or even in the cytoplasm. Differentiating ILC from IDC may be significant for the determining the possible prognostic consequences, as it has been suggested that ILC sets its own distinguishable features like presenting at an older age, with considerably larger tumors, and a tendency for displaying multifocality, multicentricity and chances of involvement of bilateral breast as well. The follow-up measures taken by two large studies have shown that the prognosis for patients with ILC may be similar to IDC early on, or even better, but after 10 years, the risk of recurrences and distant metastases, increase further $[17 ; 18]$.

The negative implications of the loss of Ecadherin expression have been studied in many reports, also the different alterations in E-cadherin expression. Denoting that in quite a few cancer types, including breast cancer, those alteration are related to certain pathological features such as poor differentiation, growth pattern, and low survival rate $[22 ; 23]$.

It is this reduced expression of E-cadherin that can be regarded as one of the main molecular events due to E-cadherin role in maintaining the pathways over junctional structures, in which exchange of many aberrant signals would lead to a sequence of malignant transformation affecting the clinical outcomes of breast cancer such as tumor size, tumor grade and rate of node positivity or metastasis $[24 ; 25]$.

In our study, the cases which showed mammary cancer with lobular morphology and showed reactivity to E-cadherin, were followed, after mastectomy, and all showed multiple foci of pure ductal differentiation, and so there was consensus on the fact that the appearance of lobular pattern was most probably the result of the extensive desmoplastic stroma present in those lesions.

\section{Conclusions}

The majority of ductal carcinomas expressed cytoplasmic E-cadherin, whereas all lobular carcinomas lacked expression of E-cadherin. Only 1 (4.7\%) of 100 invasive breast carcinomas persisted to be mixed ductal and lobular carcinoma. The diagnostic utility of Ecadherin is significant in discrimination between the two major types of mammary cancers (lobular and ductal).

\section{Recommendations}

Further study is required on the correlation with staging, hormonal status and HER2 expression of E-cadherin positive tumors and its implication on the prognosis. The analysis would better be repeated after excluding all ILCs, on the basis that previous data have shown that ILCs are E-cadherin negative irrespective of their grade.

\section{Acknowledgement}

To all members of histopathology department in Hawler Medical University/ college of medicine. And staff of Maternity Hospital, Erbil for collecting specimens of tissue samples. Thanks are also due to Dr. 
Nazdar Al-khateeb for her constant motivation and assistance.

\section{Conflict of interest}

There was no conflict of interest regarding the publication of this article.

\section{References}

[1]Lokhetty D, White VA, Watanabe R, Cree.WHO Classification of Tumours, 5thed. International Agency for Reasearch on Cancer (IARC); 2019.

[2]Yang L, Wang X, Zhu L, Wang H, Wang B, Zhao Q et al. Significance and prognosis of epithelial cadherin expression in invasive breast carcinoma. Oncology Letters 2018; 16: 1659-1665. doi: 10.3892/ol.2018.8836.

[3]Hasan, B. Erbil Cancer Registry Annual Data Announcement for the Year 2018.

[4]Turashvili G, Bouchal J, Burkadze G, Kolář Z. Differentiation of tumors of ductal and lobular origin: I. Proteomics of invasive ductal and lobular breast carcinomas. Biomed. Papers 2005; 149(1), 57-62.

[5]Chen Z, Yang J, Li S, Lv M, Shen Y, Wang $B$ et al. Invasive lobular carcinoma of the breast: A special histological type compared with invasive ductal carcinoma. PLoS ONE 2017 12(9). doi.:10.1371/journal. pone.0182397.

[6]Acs G, Lawton TJ, Rebbeck TR, Livolsi VA, Zhang PJ. Differential expression of Ecadherin in lobular and ductal neoplasms of the breast and its biologic and diagnostic implications. Am J Clin Pathol 2001; 115:85-98.

[7]Zaha DC. Significance of immunohistochemistry in breast cancer.
World J Clin Oncol 2014; 5(3): 382-392 doi: 10.5306/wjco.v5.i3.382.

[8]Dhanya AN, Niranjana Murthy B. Role of E-cadherin expression in breast carcinoma. The Journal of Medical Sciences 2018; 4(2): $38-41$.

[9]Chekhun S, Bezdenezhnykh N, Shvets J, Lukianova N. Expression of biomarkers related to cell adhesion, metastasis and invasion of breast cancer cell lines of different molecular subtypes. Exp Oncol 2013; 35(3): 174-179.

[10]Bassarova A, Torlakovic E, Sedloev T, Hristova S, Trifonov D and Nesland J. Simultaneous bilateral breast carcinoma: Histopathological characteristics and CD44/catenin-cadherin profile. Histol Histopathol 2005; 20: 791-799. doi:10.14670/HH-20.791.

[11]Turashvili G, Bouchal J, Baumforth K, Wei W, Dziechciarkova $M$ et al. Novel markers for differentiation of lobular and ductal invasive breast carcinomas by laser microdissection and microarray analysis. BMC Cancer 2007; 7(1):55. doi: 10.1186/BMC-2007.2407.

[12]Suciu C, Cîmpean A, Mureşan A, Izvernariu D, Raica D. E-cadherin expression in invasive breast cancer. Romanian Journal of Morphology and Embryology 2008; 49(4):517-523.

[13]Borcherding N, Cole K, Kluz P, Jorgensen M, Kolb R, Bellizzi A et al. Reevaluating E-Cadherin and B-Catenin a pancancer proteomic approach in breast cancer. Am J Clin Pathol 2018; 188(8): 1910-1920. doi: 10.1016/j.ajpath.2018.05.003. 
[14]Esposito N, Chivukula M, Dabbs DJ. The ductal phenotypic expression of the Ecadherin/catenin complex in tubulolobular carcinoma of the breast: an immunohistochemical and clinicopathologic study. Modern Pathology 2007; 20, 130-138. [15]Qureshi HS, Linden MD, Divine, Raju UB. E-Cadherin status in breast cancer correlates with histologic type but does not correlate with established prognostic parameters. Am J Clin Pathol 2006; 125(3): 377-385

[16]Younis L, Sakka H, Haque I. The prognostic value of E-cadherin expression in breast cancer. International Journal of Health Sciences 2007; 1(1).

[17]Pai K, Baliga P, Shrestha BL. ECadherin expression: A diagnostic utility for differentiating breast carcinomas with ductal and lobular morphologies. Journal of Clinical and Diagnostic Research 2013; 7(5): 840-84. doi: 10.7860/JCDR/2013/5755.2954

[18]Zhao L, Yang X, Khan A, Kandil D. Diagnostic role of immunohistochemistry in the evaluation of breast pathology specimens. Arch Pathol. Lab Med 2014; 138(1).

[19] Singhai R, Patil V, Jaiswal S, Patil S, Tayade M. E-Cadherin as a diagnostic biomarker in breast cancer. N Am J Med Sci 2011; 3(5): 227-233. doi: 10.4297/najms2011.3227

[20]Cowen P, Hastell S. Cadherins and catenins in breast cancer. Current Opinion in Cell Biology 2005; 17(5): 499-500.

[21]Goldstein NS. Does the level of Ecadherin expression correlate with the primary breast carcinoma infiltration pattern and type of systemic metastasis? Am J Clin Pathol 2002; 118: 425-434.

[22]Mir MR, Shabir N, Wani KA, Shaff A, Hussein I, Banday $M$ et al. Association between p16, hMLH1 and E-cadherin promoter hypermethylation and intake of local hot salted tea and sun-dried foods in Kashmiris with gastric tumors. Asian Pac J Cancer Prev 2012; 13(1): 181-186. [23]Yuan Y-L, Wang Y-M, Liu H, Qin GF, Tang AG, Duan Y. Aberrant expression of ecadherin in lung tissues of patients with probable lung cancer. Asian Pac J Cancer Prev 2012; 13(10): 5149-53.

[24] Asiaf A, Ahmad ST, Aziz SA, Malik AA, Rasool Z, Masood A et al. Loss of Expression and Aberrant Methylation of the CDH1 (E-cadherin) Gene in Breast Cancer Patients from Kashmir. Asian Pac J Cancer Prev 2014; 15 (15), 6397-6403. doi: 10.7314/APJCP. 2014.15.15.6397.

[25]Popescu CI, Jiuşcă SA, Liliac L, Avadanei R, Ceauşu R, Cîmpean AM et al. E-cadherin expression in molecular types of breast carcinoma. Rom J Morphol Embryol 2013; 54(2): 267-273. 